\title{
Pancreatic Lipase Measurement
}

National Cancer Institute

\section{Source}

National Cancer Institute. Pancreatic Lipase Measurement. NCI Thesaurus. Code C117841.

The determination of the amount of pancreatic lipase present in a sample. 